\title{
Quantum backflow effect and nonclassicality
}

\author{
Francesco Albarell: f and Tommaso Guaita \\ Quantum Technology Lab, Dipartimento di Fisica, Università degli Studi di Milano, I-20133 Milano, Italy \\ Matteo G. A. Pari周 \\ Quantum Technology Lab, Dipartimento di Fisica, Università degli Studi di Milano, I-20133 Milano, Italy and \\ INFN, Sezione di Milano, I-20133 Milano, Italy
}

(Dated: July 3, 2018)

\begin{abstract}
The quantum backflow effect is a counterintuitive behavior of the probability current of a free particle, which may be negative even for states with vanishing negative momentum component. Here we address the notion of nonclassicality arising from the backflow effect, i.e. from the negativity of the probability current, and analyze its relationships with the notion of nonclassicality based on the negativity of the Wigner function. Our results suggest that backflow is linked to a different, and in fact more restrictive, notion of nonclassicality, the negativity of the Wigner function being only a necessary prerequisite for its occurrence. This hierarchical structure may be confirmed by looking at the addition of thermal noise, which more easily destroys the negativity of the probability current than the negativity of the Wigner function itself.
\end{abstract}

PACS numbers: 03.65.-w

\section{INTRODUCTION}

The so-called quantum backflow effect consists of a counterintuitive behavior of the probability current of a quantum free particle in one dimension: the current may assume negative values even for wave-packets without negative momentum components. This means that for states with a wavefunction with only positive momenta, the probability of remaining in a certain region, e.g. $x<x_{0}$, may increase with time. This effect was earlier discovered in connection with the discussion of the arrival-time problem in quantum mechanics [1], but it was studied in details only few years later [2]. In particular, a bound for the maximal fraction of the probability that can flow backwards during a finite time interval was found. This bound, given by the adimensional constant $c_{b m} \approx 0.04$, is independent from the mass of the particle and from the duration of the effect itself. Remarkably, this value is also independent on the Planck constant $\hbar$, thus suggesting backflow as an independent quantum effect.

More recently, the backflow effect has attracted some more attention [3, 4]: improvements in the numerical estimation of $c_{b m}$ have been addressed [5, 6] and additional bounds, analytic examples, and connections with realistic measurements have been provided [7-9]. Finally, an explicit scheme to detect backflow in a Bose-Einstein condensate has been proposed [10]. The effect was found also for a particle in a linear potential [11] and for a Dirac particle [12]. An analogue effect for angular momentum has been studied as well [13].

The backflow effect is an intrinsically quantum phenomenon, for which there is no classical analogue [14]. It is intimately connected with the fact that quantum-mechanical distributions in the phase space, e.g. the Wigner function [15, 16], are not always positive functions and thus to the idea of negative probability in quantum mechanics [17, 18]. On

\footnotetext{
*francesco.albarelli@unimi.it $\dagger \overline{\text { matteo.paris@ fisica.unimi.it }}$
}

the other hand, the backflow effect appears in connection with propagation of a quantum particle and thus it cannot be entirely traced back to noncommutativity of the quantum phase space, i.e. to static nonclassicality revealed by negativity of the Wigner function. At the same time, despite being a dynamical effect, the occurrence of backflow is entirely determined by the properties of the initial quantum state since, as we will see, it occurs for systems where the dynamics in the phase space is essentially classical.

In the quantum statistical description of physical systems, the fact that quasiprobability distributions in the phase space may assume negative values is strongly linked to the notion of nonclassical states, as well as to the quantification of such nonclassicality [19-32]. In turn, the main goal of this paper is to investigate whether and how the backflow of probability, i.e. negativity of the probability current, is connected, either quantitatively or qualitatively, to the notion of nonclassicality stemming from negativity of the Wigner function, i.e. from phase space analysis. Our results indicate that quantum backflow pinpoints a different, more restrictive, notion of nonclassicality, with the negativity of the Wigner function being only a necessary prerequisite for the occurrence of backflow. This picture is confirmed by looking at the effect of noise, which more easily destroys the negativity of the probability current than the negativity of the Wigner function itself.

The paper is structured as follows. In Section $\Pi$ we review the phase space description of dynamics in quantum mechanics and introduce the backflow effect from this point of view. We also review the volume of the negative part of the Wigner function as a quantifier of nonclassicality, and briefly explore the general relationship between the two concepts. In Section III. we explicitly explore the connection between negativity of probability current and negativity of the Wigner function for states expressed as a superposition of two Gaussian wave packets. In Section IV] we analyze the behavior of the backflow in the presence of noise, i.e. under the operation of Gaussian smoothing of the Wigner function, and prove explicitly that negativity of the Wigner function is more robust against 
noise than negativity of the probability current. Section $\mathrm{V}$ closes the paper with some concluding remarks.

\section{PHASE SPACE DYNAMICS AND QUANTUM BACKFLOW EFFECT}

\section{A. Phase Space Dynamics}

A pure quantum state of a particle moving along a line (coordinate denoted by $x$ ) may be described by its wave function in the position representation $\psi_{t}(x)=\left\langle x \mid \psi_{t}\right\rangle$. A fully equivalent representation may be also given in terms of a phase space distribution function. In fact, the so-called Wigner function [15] contains the full information about the state of the system. For a pure state the Wigner function is given by

$$
\begin{aligned}
W(x, p ; t) & =\frac{1}{2 \pi} \int \mathrm{d} y \psi_{t}^{*}\left(x+\frac{1}{2} y\right) \psi_{t}\left(x-\frac{1}{2} y\right) e^{\mathrm{i} p y}, \\
& =\frac{1}{2 \pi} \int \mathrm{d} q \phi_{t}^{*}\left(p+\frac{1}{2} q\right) \phi_{t}\left(p-\frac{1}{2} q\right) e^{-\mathrm{i} x q},
\end{aligned}
$$

where the first line is the expression in terms of the position wave function $\psi_{t}(x)$ and the second one is the equivalent momentum representation, $\phi_{t}(p)=(2 \pi)^{-1 / 2} \int \mathrm{d} x e^{-i p x} \psi_{t}(x) \equiv$ $\left\langle p \mid \psi_{t}\right\rangle$ being the momentum representation of the wave function. The Wigner function is a real valued function, bounded by $|W(x, p ; t)| \leq \frac{2}{\pi}$ and normalized. On the other hand, it may take negative values and thus it cannot be interpreted as a probability distribution in the phase space.

For systems subject to a potential depending only on the coordinates, i.e. governed by the Hamiltonian

$$
H=\frac{p^{2}}{2 m}+U(x)
$$

the Wigner function obeys the continuity equation

$$
\frac{\partial}{\partial t} W(x, p ; t)+\operatorname{div} \mathbf{J}=0,
$$

where

$$
\mathbf{J}=\left(\begin{array}{c}
J_{x} \\
J_{p}
\end{array}\right)
$$

is the Wigner function flow of the system in the phase space [14, 33-35]. This Wigner flow can be decomposed as the product $\mathbf{J}=W \mathbf{v}$, where $\mathbf{v}=\mathbf{J} / W$ may be interpreted as the velocity of the phase space flow. Remarkably, for potentials at most quadratic in $x$, the velocity field $\mathbf{v}$ coincides with its classical analogue

$$
\mathbf{v}=\left(\begin{array}{c}
\dot{x} \\
\dot{p}
\end{array}\right)=\left(\begin{array}{c}
\partial_{p} H \\
-\partial_{x} H
\end{array}\right)
$$

For this class of potentials the flow is thus Liouvillian, i.e. $\operatorname{div} \mathbf{v}=0$, and the Wigner function flows in the phase space as an incompressible fluid.
Some typical quantum effects arise as a consequence of the fact that the Wigner function can take negative values. E.g. it can be easily seen that in the regions where $W$ is negative the Wigner flow $\mathbf{J}=W \mathbf{v}$ takes place in the direction opposite to the velocity $\mathbf{v}$, which, as we have seen, gives the direction of the classical phase space flow.

\section{B. The quantum backflow effect}

The properties illustrated in the previous Section may give rise to somewhat surprising results, such as the so-called quantum probability backflow effect. Let us consider a one dimensional free particle, whose initial state is a wave packet containing only components of positive momentum. Its wave function at time $t=0$ is given by

$$
\psi(x, 0)=\frac{1}{\sqrt{2 \pi} \hbar} \int_{-\infty}^{+\infty} \mathrm{d} p e^{\frac{i p x}{\hbar}} \phi(p)
$$

where $\phi(p)$ is a function which vanishes for negative values of $p$. In this situation the Wigner function of the particle is entirely localized in the positive momentum half plane of the phase space.

As we have seen the Wigner flow for a free particle coincides with the classical phase space flow, that is the one given by the velocity

$$
\mathbf{v}=\left(\begin{array}{c}
\frac{p}{m} \\
0
\end{array}\right)
$$

In the positive momentum region, where our particle is localized, the velocity is therefore always in the positive $x$ direction. However, in points where the Wigner function takes negative values, the Wigner flow points in the negative $x$ direction. Let us, in particular, consider the total Wigner volume found in the $x \geq 0$ half plane in phase space. This is given by

$$
\int_{-\infty}^{+\infty} \mathrm{d} p \int_{0}^{+\infty} \mathrm{d} x W(x, p ; t)
$$

and it coincides with the probability of finding the particle in the positive position semi-axis at a given time, that is

$$
P(t)=\int_{0}^{+\infty} \mathrm{d} x\left|\psi_{t}(x)\right|^{2}
$$

By the continuity equation (4), the time derivative of this volume is given by the Wigner flow through the $x=0$ line in phase space:

$$
j(t):=\frac{d}{d t} P(t)=\int_{-\infty}^{+\infty} \mathrm{d} p \frac{p}{m} W(0, p ; t) .
$$

The expression in Eq.(11) coincides with the quantum mechanical probability current in the origin, i.e.

$$
j(t)=\frac{i \hbar}{2 m}\left(\psi_{t}(0) \frac{\partial \psi_{t}^{*}}{\partial x}(0)-\psi_{t}^{*}(0) \frac{\partial \psi_{t}}{\partial x}(0)\right) .
$$


According to classical intuition, one would expect the wave packet described above to move in the positive spatial direction with a constant average velocity and hence the probability $P(t)$ to increase monotonically with time, as the particle moves into the positive position semi-axis. However, this is the case only for states which mimic classical behavior sufficiently well, i.e. states whose Wigner function is always positive. Conversely, if the Wigner function takes negative values, its phase space flow can be in the negative direction even in the positive momentum region and therefore, if this negative flow occurs in a sufficiently large section of the $x=0$ line, the derivative (11) can indeed take negative values.

As a consequence, for a generic quantum state, even if in the limit $t \rightarrow+\infty$ the probability $P(t)$ globally and monotonically increases, approaching the limiting value $P(t)=1$, there may exist time intervals in which it is a locally decreasing function of time. The particle thus appears to return towards the negative semi-axis. In order to quantify the backflow effect, one may consider the maximum amplitude of such temporary decrease of the probability density, i.e.

$$
\beta[\psi]:=\left|\inf _{t_{1}<t_{2}}\left[P\left(t_{2}\right)-P\left(t_{1}\right)\right]\right| .
$$

The increase in probability over a time interval $\left(t_{1}, t_{2}\right)$ (the most negative values of which we must find to compute backflow) can be expressed in terms of the phase space flow in Eq. (11) as follows

$$
F\left(t_{1}, t_{2}\right):=P\left(t_{2}\right)-P\left(t_{1}\right)=\int_{t_{1}}^{t_{2}} \mathrm{~d} t j(t) .
$$

Upon considering the incompressible fluid nature of the Wigner flow, one may define a natural motion of phase space points so that this motion has velocity given by the field $\mathbf{v}$ : a point initially in $(x, p)$, after a time interval $t$ is mapped to

$$
\varphi_{t}(x, p)=\left(\begin{array}{c}
x+\frac{p}{m} t \\
p
\end{array}\right) .
$$

Because of the incompressible nature of the flow, the Wigner density remains constant along this motion, that is

$$
W(x, p ; t)=W\left(\varphi_{-t}(x, p) ; 0\right) .
$$

Using this result we can express function (14) as

$$
\begin{aligned}
F\left(t_{1}, t_{2}\right) & =\int_{\mathcal{R}} \mathrm{d} x \mathrm{~d} p W\left(x, p ; t_{2}\right)-\int_{\mathcal{R}} \mathrm{d} x \mathrm{~d} p W\left(x, p ; t_{1}\right) \\
& =\int_{\varphi_{-t_{2}}(\mathcal{R})} \mathrm{d} x \mathrm{~d} p W(x, p ; 0)-\int_{\varphi_{-t_{1}}(\mathcal{R})} \mathrm{d} x \mathrm{~d} p W(x, p ; 0) \\
& =\int_{\Omega} \mathrm{d} x \mathrm{~d} p W(x, p ; 0)
\end{aligned}
$$

where $\mathcal{R}$ is the $x \geq 0$ half-plane and the region $\Omega=\varphi_{-t_{1}}(\mathcal{R}) \backslash$ $\varphi_{-t_{2}}(\mathcal{R})$ is an angular sector in the phase space. In polar coordinates $\Omega$ is defined by

$$
\frac{\pi}{2}+\arctan \left(\frac{t_{1}}{m}\right) \leq \phi \leq \frac{\pi}{2}+\arctan \left(\frac{t_{2}}{m}\right),
$$

and no constraint on the radial coordinate. The increase in probability over the time interval $\left(t_{1}, t_{2}\right)$ may be thus seen as the flow of the Wigner volume initially (at $t=0$ ) in the region $\Omega$ into the $x \geq 0$ half-plane. If there exists at time $t=0 \mathrm{a}$ sector $\Omega$ in which the Wigner function has negative integral, then there is also a time interval in which this probability increase is actually negative and the state shows the backflow effect. See [36] for a detailed analysis of integrals of the Wigner function on angular sectors in phase spaces.

\section{Quantum backflow and nonclassicality}

The backflow effect cannot be observed for a particle moving according to the classical laws of motion. In this sense its occurrence is a manifestation of the genuine quantum nature of the state under investigation. In the previous Section, we have seen how negativity of the Wigner function is a prerequisite to observe negativity of the probability current, and a question arises about the general connection between the two notions of nonclassicality.

A common approach to the notion of nonclassicality involves the noncommuting nature of quantum canonical variables, which implies the existence of an entire family of $s$ ordered quasidistributions in the phase space [19]. Singularity, or negativity, of one or more quasidistributions implies that the methods of classical statistics fail to describe the properties and the phenomenology of a given state, and is thus taken as a signature of nonclassicality [20]. The most fundamental definition of nonclassicality is based on the GlauberSudarshan $P$ function, whereas negativity of the Wigner function, besides being measurable experimentally [37-41], captures the nonclassical features involved in quantum interference [42] and has been recognized as a resource for quantum computation [43-45]. More generally, the presence of negative values in the Wigner function of a quantum state have been recognized as a sufficient condition for nonclassicality. In particular, the volume of the negative part has been introduced as a nonclassicality measure [46].

We will use the actual volume of the negative part (not its double or its normalized versions, sometimes used in the literature), which is defined as

$$
\Delta[\psi]=\frac{1}{2} \iint \mathrm{d} x \mathrm{~d} p[|W(x, p ; t)|-W(x, p ; t)] .
$$

If we choose $t_{1}$ and $t_{2}$ as the time interval corresponding to the minimum in Eq. [13), then $-F\left(t_{1}, t_{2}\right)$ is equal to the backflow measure of the state $\beta[\psi]$. In this way we may identify the Wigner negativity volume $\Delta$ as an upper bound to the backflow: if we denote by $V_{\Omega}^{+}\left(V_{\Omega}^{-}\right)$the volume of the positive (negative) part of the Wigner function on the sector $\Omega$ then, recalling equation (17), we may write the following inequality

$$
\beta[\psi]=-\left(V_{\Omega}^{+}-V_{\Omega}^{-}\right) \leq V_{\Omega}^{-} \leq \Delta .
$$

This confirms that nonclassicality as defined by Eq. (21) is a necessary condition for backflow. Moreover, a question arises 
on whether a more precise quantitative relation exists between $\beta$ and $\Delta$. In order to check whether this is the case, we consider an explicit example and analyze in some details the two quantities for superpositions of Gaussian wave-packets.

\section{SUPERPOSITIONS OF GAUSSIAN STATES}

\section{A. Quantum backflow for superpositions of Gaussian States}

The quantum backflow effect is not observed in states with a sufficiently classical behavior, such as those with a Gaussian wave-function. However, it may easily arise by taking quantum superpositions of such semiclassical states, which provide a natural case study. In particular, we are going to consider the superposition of two Gaussian momentum wave-packets of width $\sigma$ centered on different positive momenta. An overview of quantum backflow for such states may be found in [8]. For $\sigma \rightarrow \infty$ one recovers a superposition of two plane waves with different momenta, which is the simplest example of a state presenting backflow [2, 47], though it does not correspond to a physical state. In the following, we analyze the backflow for a general normalized superposition with complex coefficients of two Gaussian wave packets. These state are an example of the Gaussian cat states [48], introduced as a generalization of the so-called cat states often studied in quantum optics [49, 50].

Our focus is not on a systematical analysis of the effect in the whole range of physical parameters. Rather, our main goal is to compare backflow and nonclassicality in some relevant settings. To this aim, we are interested in finding a state which gives a local (in the parameter space) maximum of the backflow and to study the states in the neighboring region of the parameter space.

Upon switching to natural units (i.e. $\hbar=1$ ) and choosing a particle with unit mass, $m=1$, we consider states with the following initial momentum representation

$$
\phi_{0}(p)=N\left[e^{-\left(p-p_{0}-\delta\right)^{2} \sigma^{2}}+\alpha e^{i \theta} e^{-\left(p-p_{0}\right)^{2} \sigma^{2}}\right]
$$

where all the parameters are real numbers. The normalization condition fixes the value of $N$ in terms of the other parameters as follows

$$
N\left(\sigma, p_{0}, \delta, \alpha, \theta\right)=\left(\frac{2 \sigma^{2}}{\pi}\right)^{\frac{1}{4}}\left(1+\alpha^{2}+2 e^{-\frac{1}{2} \delta^{2} \sigma^{2}} \alpha \cos \theta\right)^{-\frac{1}{2}}
$$

The time evolved wave function, its expression in position representation and the time dependent probability current in the origin can be calculated analytically, but their expressions are somewhat cumbersome and not particularly instructive: we are not reporting their explicit expressions here. One can see, however, that these quantities can be more conveniently expressed in terms of the following rescaled adimensional parameters:

$$
\tilde{p}_{0}=\sigma p_{0} \quad \tilde{t}=\frac{t}{\sigma^{2}} \quad \tilde{\delta}=\sigma \delta
$$

With this choice, the current $j(\tilde{t})$ can be expressed as the product of a dimensional factor $\frac{1}{\sigma^{2}}$ with an adimensional oscillating function of the remaining parameters $\tilde{j}\left(\tilde{t} ; \tilde{p}_{0}, \tilde{\delta}, \alpha, \theta\right)$. Upon applying a change of variables to the integral in Eq. 14 we obtain:

$$
F\left(t_{1}, t_{2}\right)=\int_{\tilde{t}_{1}}^{\tilde{t}_{2}} \mathrm{~d} \tilde{t} j(\tilde{t})
$$

with $\tilde{t}_{k}=\sigma^{2} t_{k}, k=1,2$, from which it is apparent that the width $\sigma$ only changes the size of the time interval in which backflow is observed, while the value of the backflow itself only depends on the adimensional parameters $\tilde{p}_{0}, \tilde{\delta}, \alpha$ and $\theta$. This is in agreement with Ref. [2], where it is emphasized that the duration of the backflow effect can be changed arbitrarily. However, this extra degree of freedom may be useful if we want to consider states at fixed energy. Indeed, if we want to maximize the backflow at fixed energy $E$, we can minimize the flux 26 as a function of $\tilde{p}_{0}, \tilde{\delta}, \alpha$ and $\theta$, and then choose the appropriate value of $\sigma$ to obtain a state with a given value of energy $E$.

Of course these states do not strictly fulfill the requirement of not containing negative momenta. On the other hand, the total volume of the wave function localized on the negative semi-axis in momentum representation can be arbitrarily reduced by taking a Gaussian centered on a positive momentum sufficiently larger than its width. Indeed, by taking in only values of $\tilde{p}_{0}$ larger than 3 , the negative volume is of the order of $10^{-9}$, a value corresponding to irrelevant effect on the backflow (an explicit numerical check has been performed).

Fig. 1 shows the probability $P(\tilde{t})$ and current $\tilde{j}(\tilde{t})$ for a given superposition of two Gaussian wave-packets. As it is apparent from the plot, the time intervals where probability decreases coincide with the negative regions of the current. According to Eq. (14) and since the probability $P(\tilde{t})$ is known analytically, the backflow may be easily computed if we know the time interval which contains the most negative peak of the current. However, this involves finding the zeros of the current and this should be performed numerically. Otherwise, the backflow may be also computed through a numerical integration of the negative part of the current $\frac{1}{2}(|\tilde{j}(\tilde{t})|-\tilde{j}(\tilde{t}))$ over an interval containing the most negative peak. This method does not require the exact knowledge of the zeros, though it requires to check that only the right peak is contained within the integration interval.

We now proceed to analyze the behavior of the backflow as a function of the different parameters. At first, we notice that the $\beta[\psi]$ is a decreasing function of $\tilde{p}_{0}$, at any fixed set of values of the other parameters, see the left panel of Fig. 2. Maximum backflow is therefore attained by fixing $\tilde{p}_{0}$ to its lowest allowed value; as mentioned above we choose $\tilde{p}_{0}=3$ to ensure a vanishing negative momentum component. The effect of the parameter $\theta$ is that of shifting the position of negative peaks of the current along the time axis, as it may be seen in Fig. 3. Intuition suggests that maximum backflow is obtained for a current with a minimum located in $\tilde{t}=0$, i.e. $\theta=\pi$. Actually, the central peak is not always the one corresponding to the greatest backflow; nonetheless, in order to simplify our 


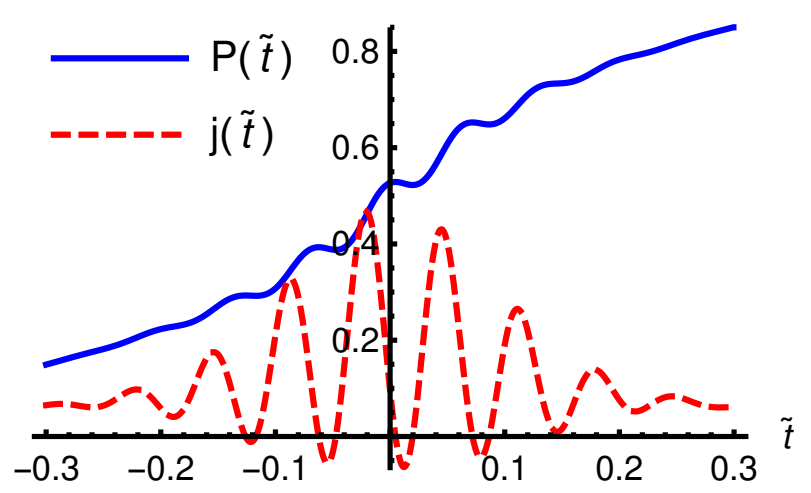

FIG. 1. (Color online) The probability $P(\tilde{t})$ (solid blue curve) and the current $j(\tilde{t})$ (dashed red curve) for a superposition of Gaussian wave-packets with $\alpha=2, \tilde{\delta}=11, \tilde{p}_{0}=3, \theta=\frac{\pi}{4}$, and $\sigma=10$. See Eq. 23) for details. The value of the current $j(\tilde{t})$ is multiplied by 10 in the figure in order to appreciate its behavior.

analysis, we focus on a parameter range for which the central peak is the most negative one.
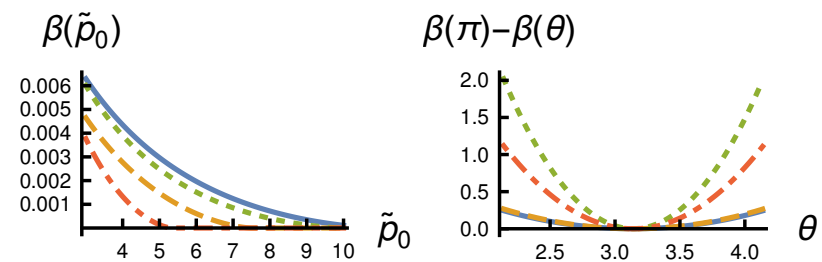

FIG. 2. (Color online) Backflow $\beta$ as a function of $\theta$ and $\tilde{p}_{0}$, the different curves represent a different choice of the couple of parameters $\alpha$ and $\tilde{\delta}: \alpha=2, \tilde{\delta}=11$ (solid blue); $\alpha=3, \tilde{\delta}=15$ (dashed orange); $\alpha=1.8, \tilde{\delta}=5$ (dotted green); $\alpha=2.5, \tilde{\delta}=8$ (dot-dashed red). Left panel: $\beta$ as a function of $\tilde{p}_{0}$, with $\theta=\pi$. Right panel: the difference between $\beta$ as a function of $\theta$ and $\beta$ obtained for $\theta=\pi$, with $\tilde{p}_{0}=3$; the values on the ordinate axis are in units of $10^{-4}$.

Unless otherwise specified, from now on we fix the values $\tilde{p}_{0}=3$ and $\theta=\pi$ and investigate the dependence of backflow on the parameters $\alpha$ and $\tilde{\delta}$. In particular, we explore the firstquadrant region of the $(\alpha, \tilde{\delta})$ plane bounded by the lines $\alpha=1$ and $\alpha=1+\tilde{\delta} / \tilde{p}_{0}$ (which is obtained by imposing $\tilde{j}(0) \leq 0$ ). For different values of $\theta$ other regions may be found where backflow is present, but no analytic expression can be found. The backflow $\beta[\psi]$ as a function of $\alpha$ and $\tilde{\delta}$ is shown in Fig. 4. We can see that $\beta[\psi]$ shows a maximum, from which it decreases going towards the boundaries of the region. The maximum is obtained for $\alpha \simeq 1.9, \tilde{\delta} \simeq 11$, corresponding to $\beta[\psi] \simeq 0.0063$ (a value slightly larger than the one found in Ref. [8]). The region closer to the value $\alpha=1$ is not shown in the plot as the backflow is not given by the central peak.

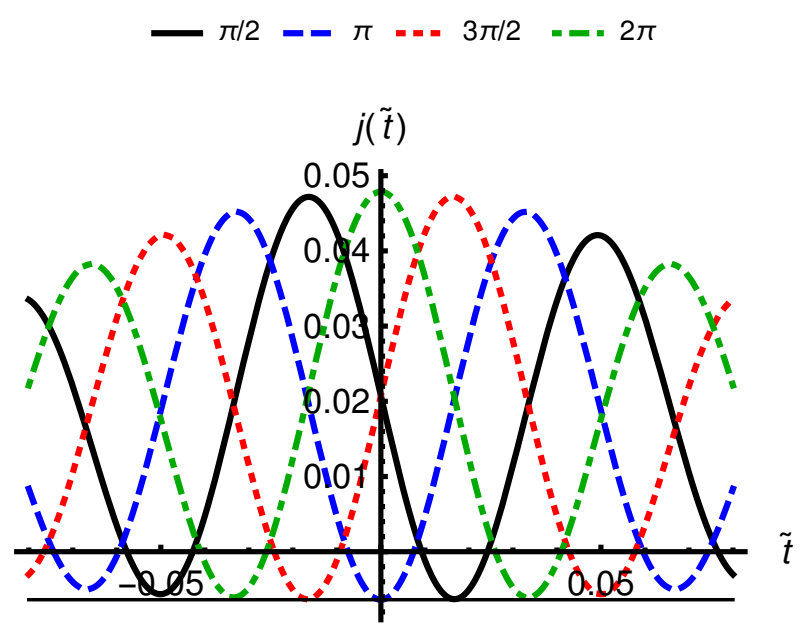

FIG. 3. (Color online) The probability current $j(\tilde{t})$ as a function of $\tilde{t}$ for different values of $\theta$ at fixed values of the other parameters ( $\alpha=2$, $\tilde{\delta}=11$ and $\tilde{p}_{0}=3$ ). The horizontal line highlights that the global minimum corresponds to the central negative peak.

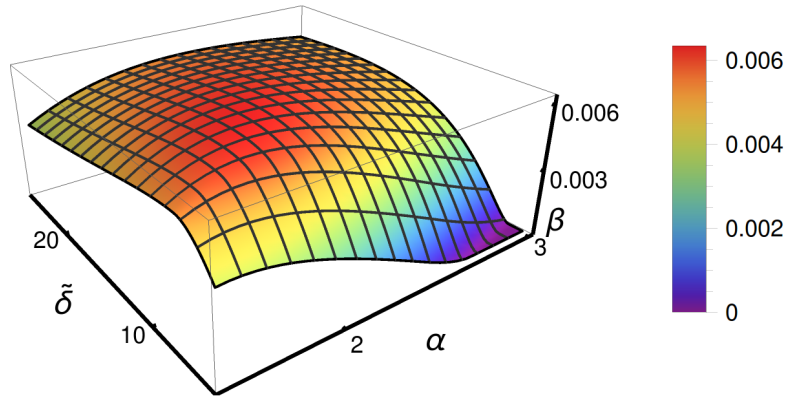

FIG. 4. (Color online) The backflow $\beta$ computed numerically as a function of parameters $\alpha$ and $\tilde{\delta}$ in the range $\alpha \in[1.5,3], \tilde{\delta} \in[5,25]$.

\section{B. Quantum backflow and Wigner nonclassicality for Gaussian superpositions}

The Wigner function of the superposition state in Eq. 23) is given by

$$
\begin{aligned}
& W_{0}(\tilde{x}, \tilde{p})=\frac{1}{\pi\left(1+\alpha^{2}+2 \alpha e^{-\frac{\tilde{\delta}^{2}}{2}} \cos \theta\right)} \cdot \\
& e^{-\tilde{x}^{2} / 2}\left[\alpha^{2} e^{-2\left(\tilde{p}-\tilde{p}_{0}\right)^{2}}+e^{-2\left(\tilde{p}-\tilde{p}_{0}-\tilde{\delta}\right)^{2}}+2 \alpha \cos (\tilde{x} \tilde{\delta}-\theta) e^{-2\left(\tilde{p}-\tilde{p}_{0}-\frac{\tilde{\delta}}{2}\right)^{2}}\right]
\end{aligned}
$$

where, consistently with Eq. [25], we used the rescaled variables

$$
\tilde{x}=\frac{x}{\sigma} \quad \tilde{p}=\sigma p .
$$

Notice that the rescaling is not altering the volume element and that $W_{0}(\tilde{x}, \tilde{p})$ does not explicitly depend on $\sigma$. This means 
that also the Wigner negativity $\Delta$, as it happens for the backflow $\beta$, does not depend on $\sigma$.

The Wigner function in Eq. (27) is characterized by two Gaussian peaks corresponding to the two momenta $p_{0}$ and $p_{0}+\delta$ and by an interference region located halfway between the two peaks. In Fig. 5 we show a contour plot of the Wigner function, which provides an intuitive explanation to the behavior of the backflow. On the one hand, the interference effects (and thus the negative regions of the Wigner function) are more pronounced if the the amplitude of the two Gaussians is the same (i.e. for $\alpha=1$ ). However, this is not leading to maximum backflow, since the Gaussian peaked at $p_{0}+\delta$ prevails in the integration region. These considerations also suggest that no monotonic relation between Wigner nonclassicality and quantum backflow may be found. As a matter of fact, since the positive parts of the Wigner function in the region $\Omega$ may compensate for the negative ones, it is possible to find states not showing backflow despite having negative Wigner function. Moreover, we may also find pairs of states with increasing backflow but decreasing negativity. This non

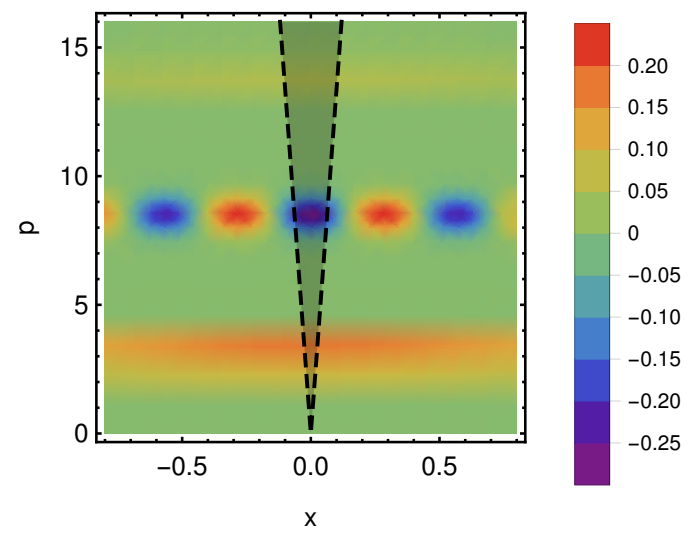

FIG. 5. (Color online) Density plot of the Wigner function of the superposition of Gaussian states with the maximum backflow, the integration region $\Omega$ is the shadowed region between the two dashed lines corresponding to $p=-\frac{m}{t_{1}} x$ and $p=-\frac{m}{t_{2}} x$.

monotonic behavior of the backflow is illustrated in Fig. 6 , where parametric plots of the backflow as a function of the Wigner negativity are shown for varying $\alpha$ or $\tilde{\delta}$.

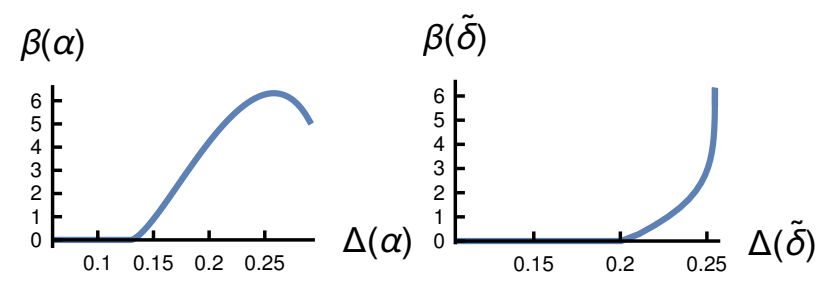

FIG. 6. (Color online) Left panel: parametric plot of the backflow $\beta$ as a function of the nonclassicality $\Delta$ for $\tilde{\delta}=11$ and varying $\alpha \in$ $[1.5,10]$. Right panel: parametric plot of the backflow $\beta$ as a function of the nonclassicality $\Delta$ for $\alpha=2$ and varying $\tilde{\delta} \in[1,20]$.

Finally, we point out that quantum backflow exhibits sud- den death for some values of the parameters. As for example, if $\alpha$ is bigger than the threshold value $\alpha=1+\tilde{\delta} / \tilde{p}_{0}$ there is no backflow. Analogue threshold values for $\tilde{\delta}$ at fixed $\alpha$ may be found. On the contrary, Wigner negativity due to the interference fringes dies only asymptotically, i.e. when a single Gaussian state is recovered. This remarkable difference may be observed in both panels of Fig. 6 , where we have regions with no backflow but nonzero Wigner negativity. In Appendix A we present the construction of a current-like quantity, for which the corresponding flux monotonically increases with the Wigner negativity. However, this quantity does depend on the state and therefore it cannot strictly represent an observable.

\section{BACKFLOW AND PHASE SPACE SMOOTHING}

We now study how robust the backflow effect is against the addition of thermal noise, corresponding to Gaussian smoothing in the phase space. We start by recalling some notions about $s$-ordered quasiprobabilities, in order to emphasize the similarity of our analysis to the notion of nonclassical depth.

\section{A. s-ordered quasiprobability distributions}

The Wigner function can be generalized to the family of $s$ ordered quasiprobability distributions [19, 51], which are routinely used in quantum statistical optics to obtain expectation values by averaging over the phase space. A quasiprobability distribution $W(x, p, s)$ is labeled by the index $-1 \leq s \leq 1$, which reflects a particular choice of the ordering of the canonical operators in the expectation value to be computed. For the specific values $s=1,0,-1$ we have the Glauber $P$ function (normal ordering), the Wigner function (symmetrical ordering) and the Husimi $Q$ function (antinormal ordering), respectively. For $s<s^{\prime}$, two quasiprobabilities of different ordering are connected through a Gaussian convolution

$$
\begin{aligned}
W(x, p, s) & =W\left(x, p, s^{\prime}\right) \star G\left(x, p, s^{\prime}-s\right) \\
& =\int \mathrm{d} x^{\prime} \mathrm{d} p^{\prime} W\left(x^{\prime}, p^{\prime}, s^{\prime}\right) G\left(x-x^{\prime}, p-p^{\prime}, s^{\prime}-s\right) ;
\end{aligned}
$$

where $\star$ denotes convolution and the function $G$ is a Gaussian defined as

$$
G(x, p, \kappa)=\frac{1}{\pi \kappa} \exp \left[-\frac{x^{2}+p^{2}}{\kappa}\right] .
$$

¿From Eq. (29) one sees that going from $s=1$ to $s=-1$ the distributions gradually become well-behaved and positive definite functions, thanks to the Gaussian smoothing. This is the idea leading to the definition of the nonclassical depth [20], which is a widely used method to quantify the amount of nonclassicality of a quantum state. Basically, this quantity is the value of $s$ closer to $s=1$ corresponding to a positive and non-singular distribution for a given state. In other words, the 
nonclassical depth represents the minimum amount of convolution needed in order to obtain a well-defined probability distribution from the $P$ function of a given state. For any given state, all the negativities in $W(x, p, s)$ must vanish for $s=-1$, as the $Q$ function is always non-negative by definition. In particular, for a pure state which is not Gaussian, we have that for all the values $-1<s \leq 0$ the quasiprobability distributions assume negative values; i.e. for such states the $Q$ function is the only distribution which is not negative [52]. This means that all non Gaussian pure states (as the superpositions of Gaussian states we have considered so far) saturate the nonclassical depth, being all maximally and equally nonclassical according to this criterion.

\section{B. s-dependent current}

Here, in order to assess the robustness of backflow against noise, we are going to consider a generalized definition of the probability current based on the $s$-ordered quasiprobability distributions. Notice that in principle only the Wigner function may be used to compute the current via Eq. (11) since the Wigner function is the only $s$-ordered quasiprobability distribution that has position and momentum probability distributions as marginals. [53] On the other hand, introducing generalized $s$-dependent currents is meaningful if we note that the convolution of a Wigner function with a Gaussian represents the Wigner function of the quantum state after the interaction with a thermal environment. Let us consider the master equation of a system interacting with a bosonic bath, expressed in terms of the canonical operators [54]

$$
\begin{aligned}
\dot{\rho}= & -\frac{\mathrm{i} \gamma}{2}(2 \bar{n}+1)([x,\{x, \rho\}]-[p,\{x, \rho\}]) \\
& -\frac{\gamma}{2}(2 \bar{n}+1)([x,[x, \rho]]+[p,[p, \rho]]),
\end{aligned}
$$

where $\gamma$ is a (small) damping coefficient and $\bar{n}$ is the average photon number of the thermal environment. In terms of the Wigner function, the solution of the above equation may be written as

$$
e^{-2 \tau} W_{t}\left(e^{-\tau} x, e^{-\tau} p\right)=W_{0}(x, p) \star G\left(x, p,-s_{\tau}\right),
$$

where $\tau=\gamma t$ and $s_{\tau}=-2(2 \bar{n}+1)\left(e^{2 \tau}-1\right)$, see e.g. [51] for details. $W(x, p, s)$ is thus the Wigner function of the state obtained from the initial one after the interaction with a noisy environment. Notice that the rescaling due to dissipation, i.e. the exponential of $\tau$ appearing on the 1.h.s. of (32), plays no role in determining the negativity of the Wigner function and the backflow.

If the initial state has the Wigner function $W_{0}(x, p)$, the state after the noisy interaction has a Wigner function given by

$$
W_{0}(x, p, s)=W_{0}(x, p) \star G(x, p,-s),
$$

where $s$ will in general be a function of the temperature, of the damping coefficient and of the interaction time. At this point, we consider $W_{0}(x, p, s)$ as the initial Wigner function of a mixed state evolving according to the free particle Hamiltonian, and we get an $s$-dependent and time dependent Wigner function

$$
W_{t}(x, p, s)=W_{0}\left(x-\frac{p}{m} t, p, s\right)
$$

which can in turn be used to compute the $s$-dependent current

$$
j(t, s)=\int_{-\infty}^{+\infty} \mathrm{d} p \frac{p}{m} W_{t}(0, p, s) .
$$

We only consider $-1 \leq s \leq 0$, in order to have a smoothing of the initial Wigner function; in terms of ordering this means going from the Wigner towards the $Q$ function.

One may wonder what happens if we exchange the order of the evolution in time and the convolution. This means convolving the time-dependent Wigner function $W_{t}(x, p)$, instead of convolving the initial one and then applying the free evolution. This way of proceeding is conceptually different and indeed yields slightly different numerical results, but the qualitative behavior is unchanged. Before proceeding we also notice that our scheme is different from considering the backflow of an open quantum system, where the expression for the probability current may be different [55].

\section{C. s-dependent backflow and negative current depth}

Having defined an $s$-dependent current we can straightforwardly apply the definition of backflow (14) and obtain an $s$-dependent backflow. As we can see in Fig. 7 the backflow vanishes for a certain $s>-1$, i.e. it exhibits sudden death, in contrast with the negativity of the Wigner function. Having more backflow initially (for $s=0$ ) usually means that the backflow of the state survives longer (i.e. it disappears for a value of $s$ closer to -1). However, as it may be seen from Fig. 7, this is not necessarily the case for any choice of the parameters.

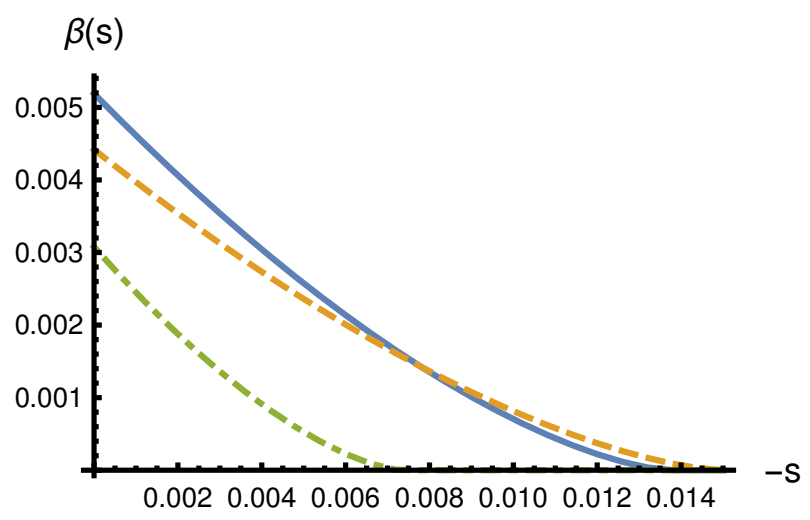

FIG. 7. (Color online) Plots of the $s$-dependent backflow as a function of the Gaussian smoothing parameter $s$. From top to bottom in the region $s \approx 0$ we have the states with $\alpha=2$ and $\tilde{\delta}=7$ (solid blue), $\alpha=2$ and $\tilde{\delta}=6$ (dashed orange), $\alpha=3$ and $\tilde{\delta}=10$ (dot-dashed green). 
In order to better analyze this behavior we introduce, in analogy with the nonclassical depth, the negative current depth, which is defined as follows. Upon denoting by $C$ the subinterval of $s \in[-1,0]$ leading to a positive $s$-dependent current in (35), then the negative current depth $s_{m}$ is defined as

$$
s_{m} \equiv \inf _{s \in C}(-s)
$$

which is a positive quantity bounded between 0 and 1 . This quantity provide an alternative quantification of backflow; instead of quantifying how much probability is flowing backwards we quantify the amount of Gaussian convolution, i.e. noise, needed to destroy the backflow effect of a given initial state.
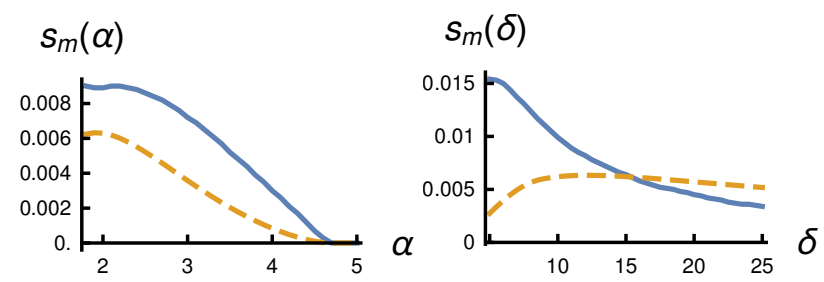

FIG. 8. (Color online) The negative current depth (solid blue) and the backflow (dashed orange). Left panel: the quantities are shown as a function of $\alpha$ for $\tilde{\delta}=11$. Right panel: as a function of $\tilde{\delta}$ for $\alpha=2$.

Fig. 8 shows that the negative current depth and the backflow of a quantum state have similar behavior, but regions where they are not monotonic exist, as it can be seen in the right panel. Upon looking at the values of $s_{m}$ in Fig. 8, and since for all these states the negativities of the Wigner function completely disappear only for $s=-1$, we conclude that the backflow is a very fragile form of nonclassicality. Notice that other criteria for nonclassicality exist and their behavior for superpositions of Gaussian states in the presence of a thermal environment has been studied [56]. Results have shown that almost all these indicators vanish for a finite Gaussian smoothing, except the visibility of the interference fringes which vanish only asymptotically.

\section{CONCLUSIONS}

The quantum backflow effect is a counterintuitive behavior observed in the probability current of a free particle, which may be negative even for states with vanishing negative momentum component. Quantum backflow may be described in the phase space, showing that its occurrence is connected to the classical phase space dynamics of a nonclassical initial state. In the case of the free particle such flow in phase space is directly connected to the probability of observing the particle in a certain region, thus it is relevant in physical problems such as determining the time of arrival. The reason for this counterintuitive behavior lies in the fact that a state with only positive momenta does not need to have a positive probability current. Since the backflow effect, despite being dynamical, is completely due to the nonclassical character of the initial state, we investigated how this kind of nonclassicality compares to the negativity of the Wigner function. In order to carry out this investigation we have focused attention to superpositions of two Gaussian states.

We found that the two notions of nonclassicality are inequivalent and the respective quantifiers do not show a monotonic behavior. We have then further characterized the backflow effect, by studying its resilience to the operation of Gaussian smoothing in phase space, which describes the interaction of the initial state with a thermal environment.

Overall, our results suggest that backflow is connected to a more restrictive notion of nonclassicality, the negativity of the Wigner function being just a necessary condition for its occurrence. Backflow has a different behavior in terms of the defining parameters of the state, in particular it vanishes for some threshold values. Moreover, the negativity of the probability current is a feature which is more easily destroyed by a thermal environment than the negativity of the Wigner function itself, confirming the idea that backflow is a nonclassical effect of a higher order and thus more fragile.

\section{Appendix A: A redefined current}

Having concluded in Section [II] that backflow is not monotonically linked to the negative Wigner function volumes, one may try to understand if these negative volumes can actually be linked to some current-like quantity. To this aim, one would have to restrict integral (11) from the whole $x=0$ axis, to the sole parts where it is crossed by the negative volumes in their phase space motion. This restriction gives the following expression:

$$
\begin{gathered}
\int_{p_{1}}^{p_{2}} \mathrm{~d} p \frac{p}{m} W_{t}(0, p)= \\
=\int_{-\infty}^{+\infty} \mathrm{d} x\left(\int_{p_{1}}^{p_{2}} \frac{1}{2 \pi} e^{i p x} \mathrm{~d} p\right) \frac{-i}{2 m}\left[\psi^{*}\left(\frac{x}{2}, t\right) \frac{\partial}{\partial x} \psi\left(-\frac{x}{2}, t\right)\right. \\
\left.+\psi\left(-\frac{x}{2}, t\right) \frac{\partial}{\partial x} \psi^{*}\left(-\frac{x}{2}, t\right)\right] .
\end{gathered}
$$

A closer examination of this expression induces us to consider a new quantity defined as

$$
\eta_{p_{1}, p_{2}}(t):=\int_{-\infty}^{+\infty} \mathrm{d} x \delta_{p_{1}, p_{2}}(x) J(x, t)
$$

where

$$
J(x, t)=\frac{i}{2 m}\left(\psi(x, t) \frac{\partial}{\partial x} \psi^{*}(x, t)-\psi^{*}(x, t) \frac{\partial}{\partial x} \psi(x, t)\right)
$$

and

$$
\delta_{p_{1}, p_{2}}(x):=\frac{1}{\pi x} \sin \left(p_{1} x\right)-\frac{1}{\pi x} \sin \left(p_{2} x\right) .
$$

This quantity (defined by parameters $p_{1}$ and $p_{2}$ which have the dimensions of momentum) has a similar structure to (A1) 
but takes real values for all times $t$. Accordingly we expect its negative flux over time to behave in a similar way to the negative volume of the Wigner function.

To give a physical interpretation of this newly defined $\eta_{p_{1}, p_{2}}$, it is interesting to observe that it is the difference of two objects, each one expressed as the convolution of the ordinary current $J(x, t)$ with a smooth approximation of the Dirac delta. Such objects can be thought to represent a probability current arising from a realistic position measurement. These measurements have a finite spatial resolution given by the widths $\frac{1}{p_{1}}$ and $\frac{1}{p_{2}}$ of the approximations of the delta used. This leads us to interpret $\eta_{p_{1}, p_{2}}$ as the difference between two realistic current measurements with different spatial resolutions given by the inverses of $p_{1}$ and $p_{2}$.

Of course the construction of this currents depends strongly on the values of parameters $p_{1}$ and $p_{2}$, which have to be chosen in such a way as to ensure that the negative volume of the state considered passes through the interval $\left(p_{1}, p_{2}\right)$ in its motion through phase space. As this choice is state dependent, the flux of $\eta_{p_{1}, p_{2}}$ cannot be considered a true observable. However, for the superpositions of Gaussians previously used, with a fixed value of $\tilde{\delta}$ and for consequently chosen values of $p_{1}$ and $p_{2}$, the negative flux of $\eta_{p_{1}, p_{2}}(t)$ shows a dependence on parameter $\alpha$ which is remarkably similar to that of the total negative volume of the Wigner function $\Delta$. This is well illustrated by Fig. 9, which shows the parametric dependence of the negative flux of $\eta_{p_{1}, p_{2}}$ on nonclassicality $\Delta$ for varying values of the parameter $\alpha$ and at fixed values of $\tilde{\delta}$. Equivalent results are found for different values of $\tilde{\delta}$.

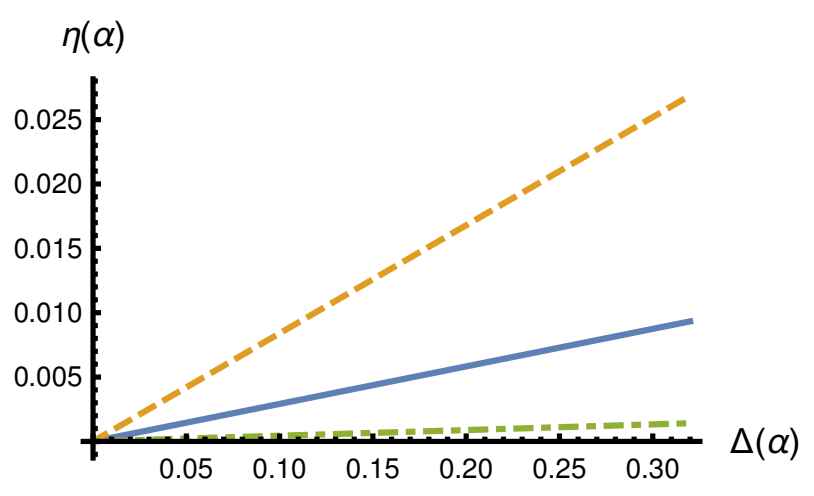

FIG. 9. (Color online) Parametric plot of the negative flow of $\eta_{p_{1}, p_{2}}$ versus the nonclassicality $\Delta$ for $\tilde{\delta}=9.5$ (dot-dashed green), $\tilde{\delta}=10$ (solid blue) and $\tilde{\delta}=10.5$ (dashed orange). The parameter $\alpha$ varies in the range $\alpha \in[0.01,5]$, while the two defining parameters of the new current are kept fixed at the values $p_{1}=7$ and $p_{2}=9$.

\section{ACKNOWLEDGMENTS}

This work has been supported by EU through the Collaborative Project QuProCS (Grant Agreement 641277) and by UniMI through the H2020 Transition Grant 15-6-3008000625.
[1] G. Allcock, Ann. Phys. 53, 253 (1969)

[2] A. J. Bracken and G. F. Melloy, J. Phys. A 27, 2197 (1994)

[3] M. V. Berry, J. Phys. A 43, 415302 (2010).

[4] P. Strange, Eur. J. Phys. 33, 1147 (2012).

[5] S. P. Eveson, C. J. Fewster, and R. Verch, Ann. Henri Poincaré 6, 1 (2005)

[6] M. Penz, G. Grübl, S. Kreidl, and P. Wagner, J. Phys. A 39, 423 (2006)

[7] J. G. Muga, J. P. Palao, and C. Leavens, Phys. Lett. A 253, 21 (1999)

[8] J. M. Yearsley, J. J. Halliwell, R. Hartshorn, and A. Whitby, Phys. Rev. A 86, 042116 (2012).

[9] J. J. Halliwell, E. Gillman, O. Lennon, M. Patel, and I. Ramirez, J. Phys. A 46, 475303 (2013)

[10] M. Palmero, E. Torrontegui, J. G. Muga, and M. Modugno, Phys. Rev. A 87, 053618 (2013).

[11] G. F. Melloy and A. J. Bracken, Found. Phys. , 505 (1998).

[12] G. F. Melloy and A. J. Bracken, Ann. Phys. 7, 726 (1998)

[13] P. Strange, Eur. J. Phys. 33, 1147 (2012)

[14] O. Steuernagel, D. Kakofengitis, and G. Ritter, Phys. Rev. Lett. 110, 030401 (2013)

[15] E. Wigner, Phys. Rev. 40, 749 (1932)

[16] M. Hillery, R. F. O’Connell, M. Scully, and E. Wigner, Phys. Rep. 106, 121 (1984)

[17] R. P. Feynman, in Quantum Implications: Essays in Honour of David Bohm, edited by B. Hiley and F. D. Peat (Methuen, London, 1987) Chap. 13, pp. 235-248.

[18] M. O. Scully, H. Walther, and W. P. Schleich, Phys. Rev. A 49,
1562 (1994)

[19] K. E. Cahill and R. J. Glauber, Phys. Rev. 177, 1882 (1969)

[20] C. T. Lee, Phys. Rev. A 44, R2775 (1991)

[21] C. T. Lee, Phys. Rev. A 45, 6586 (1992).

[22] C. T. Lee, Phys. Rev. A 52, 3374 (1995)

[23] V. V. Dodonov, J. Opt. B 4, R1 (2002).

[24] T. Richter and W. Vogel, Phys. Rev. Lett. 89, 283601 (2002).

[25] M. S. Kim, W. Son, V. Bužek, and P. L. Knight, Phys. Rev. A 65, $032323(2002)$

[26] W. Xiang-bin, Phys. Rev. A 66, 024303 (2002)

[27] E. Shchukin, T. Richter, and W. Vogel,Phys. Rev. A 71, 011802 (2005)

[28] L. Mišta and N. Korolkova, Phys. Rev. A 77, 050302 (2008)

[29] T. Kiesel and W. Vogel, Phys. Rev. A 82, 032107 (2010)

[30] S. Olivares and M. G. A. Paris, Phys. Rev. Lett. 107, 170505 (2011)

[31] R. Tatham, L. Mišta, G. Adesso, and N. Korolkova, Phys. Rev. A 85, 022326 (2012)

[32] A. Ferraro and M. G. A. Paris, Phys. Rev. Lett. 108, 260403 (2012)

[33] H. Bauke and N. R. Itzhak, (2011), arXiv:1101.2683

[34] D. Kakofengitis and O. Steuernagel, (2014), arXiv:1410.4367

[35] R. T. Skodje, H. W. Rohrs, and J. VanBuskirk, Phys. Rev. A 40, 2894 (1989).

[36] R. F. Werner, J. Phys. A 21, 4565 (1988)

[37] T. J. Dunn, I. A. Walmsley, and S. Mukamel, Phys. Rev. Lett. 74, 884 (1995)

[38] K. Banaszek and K. Wódkiewicz, Phys. Rev. Lett. 76, 4344 
(1996)

[39] G. Nogues, A. Rauschenbeutel, S. Osnaghi, P. Bertet, M. Brune, J. M. Raimond, S. Haroche, L. G. Lutterbach, and L. Davidovich, Phys. Rev. A 62, 054101 (2000).

[40] A. I. Lvovsky, H. Hansen, T. Aichele, O. Benson, J. Mlynek, and S. Schiller, Phys. Rev. Lett. 87, 050402 (2001)

[41] A. Allevi, A. Andreoni, M. Bondani, G. Brida, M. Genovese, M. Gramegna, P. Traina, S. Olivares, M. G. A. Paris, and G. Zambra, Phys. Rev. A 80, 022114 (2009)

[42] R. L. Hudson, Rep. Math. Phys 6, 294 (1974)

[43] A. Mari and J. Eisert, Phys. Rev. Lett. 109, 230503 (2012).

[44] V. Veitch, N. Wiebe, C. Ferrie, and J. Emerson, New J. Phys. 15, 013037 (2013).

[45] H. Pashayan, J. J. Wallman, and S. D. Bartlett, Phys. Rev. Lett. 115, 070501 (2015)

[46] A. Kenfack and K. Zyczkowski, J. Opt. B 6, 396 (2004)

[47] J. M. Yearsley and J. J. Halliwell, J. Phys. Conf. Ser. 442,
012055 (2013)

[48] F. Nicacio, R. N. P. Maia, F. Toscano, and R. O. Vallejos, Phys. Lett. A 374, 4385 (2010)

[49] B. Yurke and D. Stoler, Phys. Rev. Lett. 57, 13 (1986)

[50] V. V. Dodonov and V. I. Man'ko, eds., Theory of nonclassical states of light (Taylor \& Francis, London New York, 2003).

[51] S. M. Barnett and P. M. Radmore, Methods in theoretical quantum optics (Oxford University Press, Oxford, New York, 1997).

[52] N. Lütkenhaus and S. M. Barnett, Phys. Rev. A 51, 3340 (1995)

[53] Other generalized distributions in phase space that satisfy this property exist [57, 58], but they are not relevant for the topic discussed here.

[54] B. Vacchini, J. Math. Phys. 43, 5446 (2002)

[55] J. M. Yearsley, Phys. Rev. A 82, 012116 (2010)

[56] J. Paavola, M. J. W. Hall, M. G. A. Paris, and S. Maniscalco, Phys. Rev. A 84, 012121 (2011)

[57] L. Cohen, J. Math. Phys. 7, 781 (1966)

[58] P. J. Loughlin and L. Cohen, J. Mod. Opt. 50, 2305 (2003) 